\title{
Uma reflexão sobre as políticas de atendimento para as pessoas com transtorno do espectro autista
}

\section{A reflection on the policies of attendance for persons with autistic spectrum disorders}

\author{
1 Rozana Aparecida Souza \\ 1 Joseane de Almeida dos Santos \\ 1 Juliana da Silva \\ 1 Stéfany Almeida Soares
}

1 Curso de Serviço Social do Centro Universitário de Volta Redonda (UniFOA)

\section{Resumo}

O Brasil não tem dados precisos sobre o Transtorno do Espectro Autista, porém estima-se que existam cerca de dois milhões de autistas no território nacional. Contudo, apesar de numerosos, os brasileiros autistas ainda sofrem para encontrar tratamento adequado. As dificuldades residem, sobretudo, na falta de profissionais preparados para lidar com o transtorno, principalmente na rede pública. Este estudo teve por objetivo identificar e analisar as políticas de atendimento para as pessoas com autismo e construir uma reflexão sobre o trabalho do assistente social frente a essa demanda. Trata-se de uma pesquisa qualitativa, pautada na realização de um levantamento bibliográfico em livros e artigos científicos e documental em legislações e manuais relacionados ao tema. Identificou-se que a história do autismo é marcada pela falta de consenso. O Brasil apresenta um marco jurídico normativo favorável ao atendimento desse público, entretanto é necessário superar desafios, principalmente, nas políticas de saúde e de educação inclusiva. Ao assistente social, cabe uma atuação crítica que objetive a inclusão e autonomia desses sujeitos.

\section{Palavras-chave:}

Autismo. Política de atendimento. Serviço social.

\begin{abstract}
Brazil does not have precise data on Autism Spectrum Disorder, but it is estimated that there are about two million autistic children in Brazil. However, despite being many, Brazilian autistic children still have extreme difficulty to find adequate treatment. The difficulties mainly lie on the lack of professionals prepared to deal with the disorder, especially in the public network. This study aimed to identify and analyze care policies for people with autism and to build up a reflection about the social worker's task in face of this demand. It is a qualitative research, based on the accomplishment of a bibliographical survey in books and scientific articles; and also a documental survey in laws, manuals, blogs and websites related to the topic. It has been identified that the history of autism is marked by lack of consensus. Brazil has a normative legal framework in favor of this public, but it is necessary to overcome challenges, especially in health and inclusive education policies. The social worker has a critical role that aims the inclusion and autonomy of these subjects
\end{abstract}

\section{Keywords:}

Autism. Service policy. Social service.

\section{Como você deve citar?}

SOUZA, Rozana Aparecida et al. Uma reflexão sobre as políticas de atendimento para as pessoas com transtorno do espectro autista. Cadernos UniFOA, Volta Redonda, n. 40, p.95-105, agosto 2019. 
Uma reflexão sobre as políticas de atendimento para as pessoas

com transtorno do espectro autista

\section{INTRODUÇÃO}

Segundo Guimarães \& Martins (2006), Tamanaha et al. (2008) e Brasil (2013), a primeira definição de autismo como um quadro clínico ocorreu em 1943, nos EUA, pelo médico austríaco Leo Kanner.

Estima-se que o Brasil tenha cerca de dois milhões de indivíduos com Transtorno do Espectro Autista (TEA). Contudo, apesar de numerosos, os milhões de brasileiros autistas ainda sofrem para encontrar tratamento adequado (OLIVEIRA, 2015). Segundo dados do CDC (Center of Deseases Control and Prevention), órgão ligado ao governo dos Estados Unidos, existe, hoje, um caso de autismo a cada 110 pessoas. Apesar dos altos índices de autismo, foi somente em 1993 que a síndrome foi adicionada à Classificação Internacional de Doenças da Organização Mundial da Saúde (OMS).

Essa demora se deve ao fato do pouco que se sabe sobre a questão. 0 diagnóstico ainda é impreciso e nem mesmo um exame genético é capaz de afirmar com precisão a incidência da síndrome. Para Oliveira (2015), uma vez diagnosticado autista, o "paciente" e sua família enfrentam mais uma barreira: a busca pelo tratamento e atendimentos diversos que se fazem necessários (saúde, educação, assistência social etc). As dificuldades residem, sobretudo, na falta de profissionais preparados para lidar com o transtorno, principalmente na rede pública.

Em dezembro de 2012, alguns dos direitos dos autistas passaram a ser assegurados pela lei 12.764, chamada de "Política Nacional de Proteção dos Direitos da Pessoa com Transtorno do Espectro Autista" (BRASIL, 2012). A lei reconhece que os portadores de autismo têm os mesmos direitos que todos os outros sujeitos com necessidades especiais no Brasil.

Esse panorama aponta para a necessidade de investigações sobre como se apresenta a política de atendimento para pessoas autistas, como os serviços estão/deveriam estar organizados e quais profissionais estão envolvidos no atendimento a esse público e suas famílias.

Nesse cenário de pouco conhecimento sobre o autismo, aliado à situação atual da oferta de serviços de atendimento, torna-se necessário refletir sobre como o profissional de Serviço Social tem atuado junto a esse público.

Os dados ora apresentados resultam de um projeto de iniciação científica que teve por objetivo identificar e analisar as políticas de atendimento para as pessoas com autismo e construir um debate sobre o trabalho do assistente social frente a essa demanda. Foram consultados referenciais bibliográficos disponibilizados na base de dados Scielo e legislações pertinentes à temática. Optouse por realizar uma revisão crítica de estudos e legislações nacionais (publicados no período de 2006 a 2018). Apresentam-se alguns documentos internacionais dos quais o Brasil é signatário e que são necessários para o debate (publicados na década de 1990). Nos estudos levantados, ficam evidentes as dificuldades e barreiras que perpassam o atendimento à pessoa com TEA.

Este artigo pretende apresentar um debate sobre o TEA a partir de duas "grandes" políticas, Saúde e Educação, discutindo principais dilemas e desafios no atendimento de pessoas com TEA nessas áreas. E, por fim, apresentar uma reflexão sobre a atuação do profissional de Serviço Social e o autismo. 


\section{AUTISMO E A POLÍTICA DE SAÚDE}

Ao longo da história, o autismo primeiramente foi reconhecido como um distúrbio no qual as pessoas apresentavam dificuldades de comunicação e interação social. As primeiras produções acerca do tema foram feitas pelos psiquiatras Hans Asperger e Leo Kanner.

Leo Kanner, em 1943, ao pesquisar o caso de 11 crianças, observou uma diferenciação em seus comportamentos e a predominância de isolamento. Em seguida, o pediatra Hans Asperger, em 1944, fez uma pesquisa semelhante, caracterizando o caso de algumas crianças com comportamentos autistas (ONZI; GOMES, 2015).

Nas décadas de 1950-60, a psicanálise atribuía esse transtorno à falta de afeto, ou seja, o transtorno seria consequência de um déficit de afeto, principalmente materno, surgindo assim a expressão "mães de geladeiras". Com os estudos, o autismo passou a não ser mais classificado com uma perturbação emocional.

Em 1952, na primeira edição do Manual Diagnóstico e Estatístico de Transtornos Mentais (DSM), o diagnóstico do autismo estava caracterizado como uma classificação de esquizofrenia (ONZI; GOMES, 2015).

Em 2014, foi lançado o DSM-V. Nessa edição, foi definido que o desenvolvimento do autismo se apresenta até os quatro anos de idade e que vai progressivamente afetando os desenvolvimentos cognitivos e neurológicos e desaceleração do crescimento da cabeça.

Por um longo período, as definições acerca do autismo, suas causas e formas de tratamento foram marcadas por contradições, o que refletiu em diagnósticos equivocados e tratamentos inadequados, afetando a vida dos pais de crianças e jovens autistas. Segundo APA (apud ONZI; GOMES, 2015), o termo autismo perpassou por diversas alterações ao longo do tempo. Atualmente, define-se por Transtorno do Espectro Autista (TEA), conforme o Manual Diagnóstico e Estatístico de Transtornos Mentais.

O TEA, de acordo com a Classificação Estatística Internacional de Doenças (CID-10), está inserido no grupo dos Transtornos do Desenvolvimento Psicológico.

A discussão do autismo no âmbito da saúde se apresenta como um desafio: existe uma dificuldade de diálogo sobre a doença, um desafio do consenso sobre o tratamento e a necessidade de se realizar um diagnóstico precoce.

O diagnóstico precoce é essencial para o prognóstico. Esse diagnóstico pertence ao domínio dos cuidados primários da saúde, sendo necessário um arcabouço teórico que torna importante identificar sinais específicos, que ofereçam condições de diferenciar o autismo de outras patologias.

Quanto mais precoce, personalizada e intensiva se processar melhor será o prognóstico em termos de aprendizagem linguística, social, adaptativa e não menos importante, na minimização de comportamentos disruptivos (birras, agressividade, agitação, hiperatividade) que decorrem de intervenções ausentes ou desajustadas. (OLIVEIRA, 2009, p. 283).

O diagnóstico precoce se torna mais emblemático tendo em vista que o "tema do autismo implica se deparar com um contexto fortemente controverso e muitas vezes de difícil diálogo entre os psiquiatras, neurologistas, educadores, psicólogos e psicanalistas" (OLIVEIRA, 2012, p. 50). 
Uma reflexão sobre as políticas de atendimento para as pessoas

com transtorno do espectro autista

O TEA é marcado por controvérsias, pois existem diversas concepções teóricas sobre o que provoca o autismo que acabam por resultar numa falta de consenso sobre o tratamento. Porém, existe consenso sobre a concepção do autismo como uma disfunção neurológica, que clinicamente se manifesta por atraso ou desvio nas aquisições do neurodesenvolvimento e por alterações do comportamento (OLIVEIRA, 2009), que tem como sintoma principal a dificuldade de estabelecer relações afetivas e dificuldade de comunicação.

O Ministério da Saúde criou a cartilha "Diretrizes de Atenção à Reabilitação da Pessoa com TEA", objetivando orientar as equipes multidisciplinares para atender pessoas com autismo e suas famílias (BRASIL, 2013). Entre as possíveis formas de tratamento, estão terapia cognitivo comportamental, psicoterapia, musicoterapia, linguagem de sinais, análise aplicada do Comportamento, entre outras.

Segundo Bosa (2006), o tratamento é conduzido com o propósito de estimular o desenvolvimento comunicativo e social, aprimorar a capacidade de solucionar problemas, auxiliar famílias e minimizar os impactos que possam afetar a rotina dos portadores.

A família do sujeito com TEA também deve ser alvo de ações de cuidado e de orientação. Segundo Marques e Dixe (2011), após o diagnóstico, é essencial que os familiares sejam informados e orientados. A adaptação familiar exige superação das dificuldades.

Pelo fato de a etiologia do autismo ser pouco definida, os pais podem apresentar sentimentos negativos em vista da necessidade de busca por respostas. A fim de dar sentido para o que está acontecendo com seu filho, essas dificuldades enfrentadas podem gerar sentimentos confusos (SCHMIDT apud ONZI; GOMES, 2015, p.192).

O apoio das redes de proteção, amigos, familiares, profissionais de saúde e de educação é fundamental para as famílias que convivem com o TEA.

É necessário ressaltar a constituição do Sistema Único de Saúde (SUS) e o Movimento da Reforma Psiquiátrica como fatores essenciais para a melhoria do atendimento no campo da Saúde Mental - espaço prioritário para o atendimento do TEA. O SUS consolidou princípios importantes para o atendimento do TEA: a universalização da saúde, a integralidade das ações e a intersetorialidade das políticas. O Movimento da Reforma Psiquiátrica brasileira questionou a psiquiatria tradicional que pautava o atendimento dos portadores de transtornos mentais no isolamento, na contenção e na marginalizavam desse indivíduo. Esse movimento prevê um tratamento através da ótica dos direitos humanos, valorizando o indivíduo no âmbito social e a substituição dos hospitais psiquiátricos por Centros de Atenção Psicossocial (CAPS).

A população de crianças e adolescentes que sofrem com transtornos mentais é marcada por um histórico de inexistência de uma política de saúde mental infantojuvenil. Por muito tempo, essa demanda foi atendida em instituições privadas ou filantrópicas. Com a implementação do Centro de Atenção Psicossocial Infantojuvenil (CAPSi), proposto em 2002, essa realidade se alterou (COUTO et al., 2008).

O cuidado com a saúde mental infantojuvenil se deu de forma tardia e não pode ser pensada na mesma lógica que o atendimento em saúde mental para adultos.

De modo geral, as políticas de saúde mental existentes estão relacionadas aos problemas da população adulta. Na população de crianças e adolescentes, os tipos de transtorno, principais fatores de risco e de proteção, estratégias de intervenção e organização do sistema de serviços têm especificidades que não podem ser contempladas pela simples extensão das estratégias de cuidado da população adulta à população infantil e juvenil. (COUTO et al., 2008, p. 391). 
O autismo, por um tempo, esteve formalmente fora das patologias que eram atendidas na política de saúde mental. Nesse sentido, o CAPSi foi essencial na ampliação do acesso ao tratamento. Entretanto, para assegurar esse atendido digno e com qualidade, como apontam Couto et al. (2008), urge superar alguns desafios: a expansão dos serviços, ou seja, aumentar o números de dispositivos; a expansão da gestão territorial para efetividade e consolidação da política de saúde mental; ter cobertura em outros dispositivos para crianças e adolescentes; sobrecarga dos profissionais; escassez dos serviços voltados a esse público, o que dificulta o trabalho em rede.

Segundo Couto (2004), um estudo realizado pela Assessoria de Saúde Mental da Secretaria Estadual de Saúde (RJ), no início de 2000 , mostrou que, aproximadamente, $70 \%$ dos municípios utilizavam os dispositivos da rede filantrópica como principal recurso no atendimento de crianças e adolescentes com autismo no país, embora essas instituições - como APAE e Pestalozzi - tivessem anteriormente suas diretrizes voltadas exclusivamente para o atendimento de pacientes portadores de deficiência mental. Em apenas $20 \%$ dos municípios, os programas de saúde mental atendiam a casos diagnosticados de autismo e psicose, sendo o tradicional modelo ambulatorial o dispositivo utilizado para a maioria deles. Sujeitos portadores de TEA e psicoses encontram-se errantes pelas instituições, sem atendimento, e submetidos à pedagogização de suas condutas e à excessiva medicalização, com graves consequências para suas vidas.

\section{AUTISMO E A POLÍTICA DE EDUCAÇÃO}

Na política de Educação, as discussões sobre as pessoas com Transtorno do Espectro Autista (TEA) estão articuladas ao debate sobre educação inclusiva, entretanto essa inclusão deve ser alvo de reflexão.

A Constituição Federal de 1988 (BRASIL, 1988) estabelece que todas as crianças têm por direito o acesso à educação comum. Entende-se, portanto, que os sujeitos com TEA são beneficiados por esse direito, o que deve e precisa ocorrer pela educação inclusiva.

A inclusão educacional escolar no Brasil é uma ação política, cultural, social e pedagógica que visa garantir o direito de todos os alunos de estarem juntos, aprendendo e participando (BRASIL, 2008).

Segundo Nunes et al. (2013), foi a partir da Declaração Universal dos Direitos Humanos (1948) que a educação "especial" começou a ser discutida no Brasil, mas foi a partir da Constituição Federal de 1988 que se fomentou a discussão da universalização da educação, sendo implementada a educação inclusiva nas escolas regulares. Isso resultou na Política Nacional de Educação Especial na perspectiva da Educação Inclusiva (BRASIL, 2008), que designa que pessoas com deficiência, assim como as pessoas com TEA, sejam incluídas no ensino da rede regular.

Documentos, como a Declaração Mundial de Educação para Todos (ONU, 1990) e a Declaração de Salamanca (ONU, 1994), que trazem a garantia da inserção do ensino especial no ensino regular e o atendimento das necessidades de aprendizagem das crianças como objetivos, foram também marcos importantes para a discussão e expansão da educação inclusiva.

Atualmente, no cenário nacional, os marcos legais que se destacam no processo de estabelecimento de uma educação para todos são a Constituição Federal de 1988 (BRASIL, 1998) e as Leis de Diretrizes e Bases da Educação Nacional (BRASIL,1996). 
Uma reflexão sobre as políticas de atendimento para as pessoas

com transtorno do espectro autista

A Educação inclusiva é caracterizada como uma política social destinada aos alunos com necessidades educacionais especiais. Segundo a Declaração de Salamanca, o conceito de necessidades educacionais especiais deve ser amplo (BRASIL, 2012, p.17-18):

\begin{abstract}
O princípio fundamental desta linha de ação é de que as escolas devem acolher todas as crianças, independentemente de suas condições, físicas, intelectuais, emocionais, linguística e outras. Devem acolher crianças com deficiência ou bem-dotadas, crianças que vivem nas ruas e que trabalham, crianças de populações distantes ou nômades, crianças de minorias linguísticas étnicas ou culturais e crianças de outros grupos ou zonas desfavorecidas ou marginalizadas.
\end{abstract}

A educação inclusiva deve ser para todos os cidadãos, sem considerar a deficiência, cor, origem cultural ou socioeconômica, visando garantir um ambiente propício e adequado para atender as necessidades de todos os indivíduos e uma educação de qualidade. De acordo com Camargo e Bosa (2009), o ambiente e as condições adequadas são fundamentais no processo de inclusão, para que não haja prejuízos aos que acessam esse direito.

A inclusão escolar de sujeitos com TEA no ensino regular se depara com alguns desafios, como a escassa rede de apoio, pouca formação específica dos educadores e capacitação das escolas, a particularidade do diagnóstico dos alunos, além da insegurança dos pais, alunos e professores. Segundo Rodrigues et al. (2012), falta rede de apoio aos professores e educadores de alunos diagnosticados com autismo, o que amplia as dificuldades do processo de inclusão social.

Outro desafio, apontado pelos autores, é a dificuldade do trabalho pedagógico. A presença dos alunos com autismo em salas de aula regulares não determina uma inclusão imediata. Há resistências e entraves no relacionamento com outros alunos, dificuldade essa acarretada pelo próprio diagnóstico: o TEA ocasiona dificuldade de relacionamento e de comunicação.

Alunos autistas são identificados como aqueles com maior dificuldade na inclusão, o que não acontece apenas em consequência dos sintomas, mas também da falta de recursos humanos e de materiais adequados e pela dificuldade individual dos professores.

Essas dificuldades encontradas fazem com que os alunos com comportamentos "diferentes" do padrão de "normalidade" esperado sejam vistos como problemas e se tornem um desafio para os profissionais de educação. Existe, portanto, a necessidade de uma formação adequada dos profissionais e uma capacitação da escola para receber pessoas com TEA.

\begin{abstract}
É a qualidade do relacionamento professor-aluno que torna o processo educativo e a escola significativos para o educando. É preciso que os professores sejam capacitados para atender à crescente população de crianças com autismo. Infelizmente ainda é grande o número de pessoas que enxergam esses indivíduos de forma errônea e acabam não realizando a inclusão da melhor forma possível a esses alunos. Mas em todas as pesquisas estudadas, ficou clara a importância da inclusão dessas crianças em escolas regulares para promover experiências de socialização atividades diárias, tornando-as o mais independente possível. (VIEIRA et al., 2013, p.09).
\end{abstract}

Assim, a política de inclusão necessita ser aprimorada num perceptiva não só de fomentar o acesso, mas também no sentido de manter as crianças com autismo nas escolas de forma que elas, mesmo estando incluídas, não sejam excluídas por conta das dificuldades presentes nesse processo. Portanto, é essencial compreender a inclusão escolar como importante dispositivo para a organização de uma rede social que sustente a autonomia de crianças com graves transtornos (RODRIGUES et al., 2012).

Cabe salientar a existência de um ator importante na perspectiva de inclusão social das pessoas com espectro autista, que são as entidades sociais, que, na maioria das vezes, oferecem serviços visando à inclusão social. (LEITE; HETZEL, 2011). 
A Associação Brasileira do Autismo (ABRA) é uma entidade de importância, que elaborou documentos fundamentais de reivindicações de direitos das pessoas com espectro autista. Criada no dia 18 de outubro de 1988, decorrente da necessidade de existir uma associação de nível nacional que congregasse as demais associações,

a ABRA foi formalizada com o intuito de integralizar as associações existentes no país ou que venham possivelmente a existir. Tem por finalidade "a integração, coordenação e representação, a nível nacional e internacional, das entidades a ela filiadas". (LEITE; HETZEL, 2011, s/p).

A ABRA, juntamente com as associações de pais, intensificou suas ações nas políticas públicas para os autistas junto aos governos. Um marco foi o documento de 2006, definido como Metas para a década das pessoas com deficiência - Área do Autismo (ONU, 2006), que tinha como finalidade cooperar para melhoria da atenção a pessoas com TEA e outros transtornos. Esse documento foi dirigido à Secretaria Especial dos Direitos Humanos e à Coordenadoria Nacional para Integração da Pessoa Portadora de Deficiência.

É notável o papel da ABRA no processo de inclusão social, mas cabe salientar que a inclusão social é de responsabilidade maior do Estado.

\section{SERVIÇO SOCIAL E O AUTISMO: (RE)PENSAR ATRIBUIÇÕES E CONTRIBUIÇÕES}

O autismo está presente em todas as classes sociais, povos e culturas. A vida familiar sofre uma mudança drástica, tarefas cotidianas e rotineiras - tais como fazer as refeições, cuidados básicos com higiene, sono, brincadeiras, banhos de piscina, vestir e despir-se - passam a ser envoltas numa série de dificuldades que podem levar a conflitos e exaustão. A execução dessas tarefas, muitas vezes, só ocorre com vigilância intensiva (MATEUS, 2015).

É dever do Estado proporcionar para toda a população políticas públicas por meios de programas e projetos que tenham por finalidade a busca pela garantia de diretos dos indivíduos. 0 cenário jurídico normativo brasileiro, como apontado, mostra-se favorável ao atendimento do indivíduo com TEA. Além das legislações no campo da Educação, temos leis e decretos da política de Saúde e Direitos Humanos (DAMASCENO et al., 2017).

Entretanto a realidade do sujeito com TEA é marcado por inúmeras dificuldades no acesso aos seus direitos. De acordo com Amorim e Lúcio (2016), dizer ao indivíduo que ele tem direitos é insuficiente, é preciso acionar os mecanismos concretos para que esse cidadão seja inserido nos serviços. Nessa seara, os assistentes sociais, além de conhecerem e entenderem os direitos e garantias que são assegurados às pessoas com autismo, devem intervir no campo das relações sociais para concretizar a efetivação e a consolidação desses direitos, trabalhando em conjunto com a equipe na qual ele está inserido, com a família e com a sociedade.

A Lei 8.662, de 1993 (BRASIL, 2011), afirma que compete aos assistentes sociais, dentre outras ações, orientar indivíduos e grupos de diferentes camadas sociais, identificando e fazendo uso de recursos; defender seus direitos e trabalhar no planejamento, organização e administração dos benefícios e serviços sociais. Nesse sentido, mesmo se o usuário com autismo não tiver autonomia devido às especificidades de seu quadro clínico, os profissionais devem intervir, de modo que eles caminhem em busca de conquistá-la, através da socialização de informações, da inserção deles em programas e serviços e da publicização de seus direitos. 
Uma reflexão sobre as políticas de atendimento para as pessoas

com transtorno do espectro autista

\begin{abstract}
É competência do profissional de Serviço Social observar e entender as mudanças societárias e lutar pela conquista de uma sociedade mais democrática, onde haja a consolidação da cidadania e da justiça social. Ao lidar com o autismo, é indispensável que o assistente social procure compreender as especificidades dessa expressão da questão social, bem como as particularidades de cada indivíduo, tornando, assim, o seu trabalho mais qualificado, e que tenha como intervenção profissional, buscar a conscientizar as famílias sobre as políticas públicas e os direitos sociais que a criança possui, ou seja, é dever do profissional e está presente no Código de Ética, segundo (AMORIM; LÚCIO, 2016, p.07).
\end{abstract}

São vários os espaços ocupacionais em que esse profissional poderá trabalhar com crianças e adolescentes autistas: na assistência e previdência social, na educação inclusiva, nos CAPSi, dentre outros.

Segundo Damasceno et al. (2017), o assistente social é o profissional que possibilita a realização de uma aproximação sucessiva dos indivíduos com TEA com a realidade presente, entendendo as particularidades, desvelando as demandas imediatas e, assim, encontrando respostas profissionais sustentáveis.

A atuação do Serviço Social, nos casos de autismo, tem objetivo de prover proteção à vida, reduzir danos e prevenir a incidência de risco social às pessoas com TEA e aos seus familiares, fortalecendo o debate sobre a inclusão desses indivíduos no meio social, bem como na busca de eliminação de preconceito e discriminação que esse público sofre.

Segundo Oliveira (2017), há uma divergência entre o campo da reabilitação e o da atenção psicossocial. No caso do atendimento aos autistas, essa divergência tem um caráter mais político do que epistemológico ou clínico. É preciso questionar sempre: para onde as pessoas com autismo estão caminhando? Ou para onde a sociedade tem conduzido esse público?

A inclusão e a autonomia do sujeito com TEA devem ser um dos objetivos a não se perder de vista.

\title{
5 CONSIDERAÇÕES FINAIS
}

Para Guimarães e Martins (2006), especula-se que o aumento de casos de TEA seja fruto de uma reconfiguração na rede de cuidados a essa população, que teve como marco inicial a desinstitucionalização do retardo mental no final dos anos 1960, nos Estados Unidos. Tal argumento não aponta, exclusivamente, para uma substituição diagnóstica do retardo mental por autismo, mas, sobretudo, para o efeito que a rede de cuidados que se formou em torno do autismo teve, no sentido de conferir mais visibilidade a esse diagnóstico. Segundo Rios et al. (2006), não foi a epidemia que fez o autismo visível, mas a visibilidade do autismo que fez a epidemia.

"Para os portadores dessa doença, ter acesso a um tratamento adequado e educação especializada é garantir-Ihes a dignidade humana e a chance de inclusão social" (SPÍNOLA, 2014, p.61). O TEA ainda é uma doença marcada por mitos e que precisa de maior investimento na implementação de políticas públicas eficazes e eficientes no atendimento das demandas específicas de cada diagnóstico.

Segundo Spínola (2014), indivíduos com autismo precisam de políticas de saúde diferenciadas. Não basta o atendimento de psicólogos e psiquiatras: para um desenvolvimento com dignidade e para a inclusão social desse público é necessário tratamento inter e multidisciplinar, composto por um leque de profissionais, tratamento esse que deve ser custeado pelo Estado. 
Destaca-se a afirmação de Silva (2013, p.83):

uma pessoa com um transtorno, por exemplo, é, antes de tudo, uma pessoa e não um transtorno. Nesse sentido, um indivíduo com Transtorno do Espectro do Autismo não é um autista, é uma pessoa humana, nomenclatura fundamental de garantia de direitos. Um rótulo classificatório não é capaz de captar a totalidade complexa de uma pessoa, muito menos, a dimensão humana irredutível desta.

O debate sobre o autismo tem sido marcado por controvérsias e desafios. Essa situação dificulta ainda mais a vida de milhares de famílias.

\section{REFERÊNCIAS}

AMORIM, K. L. S.; LÚCIO, C. V.. A importância da atuação dos assistentes sociais para a efetivação dos direitos das crianças e dos adolescentes com autismo. $4^{\circ}$ Simpósio Mineiro de Assistentes Sociais. Belo Horizonte. p.1-14, 2016. Disponível em: http://cress-mg.org.br/hotsites/Upload/Pics/e7/e7614408cab0-4632-ac6c-8fa8ca174af9.pdf. Acesso em: 10 set. 2018.

BOSA, C.. Autismo: intervenções psicoeducacionais. Revista Brasileira de Psiquiatria. São Paulo, v. 28, suppl.1, p.:s47-s53, 2006. Disponível em: http://dx.doi.org/10.1590/S1516-44462006000500007. Acesso em: 05 maio 2018.

BRASIL, Ministério da Saúde. Secretaria de Atenção à Saúde. Departamento de Ações Programáticas Estratégicas. Diretrizes de Atenção à Reabilitação da Pessoa com Transtornos do Espectro do Autismo. Brasília: Ministério da Saúde, 2013.

BRASIL. Código de ética do/a assistente social. Lei 8.662/93 de regulamentação da profissão. Brasília: Conselho Federal de Serviço Social, 2011. Disponível em: http://www.cresses.org.br/site/index. php?option=com_content\&view=article\&id=69\&ltemid=78. Acesso em: 16 out. 2018.

BRASIL. Constituição (1988). Constituição da República Federativa do Brasil. Brasília, DF, Senado, 1998. Disponível em: http://www.planalto.gov.br/ccivil_03/constituicao/ConstituicaoCompilado.htm. Acesso em: 18 out. 2018.

BRASIL. Lei n ${ }^{10.764}$, de 27 de dezembro de 2012. Institui a Política Nacional de Proteção dos Direitos da Pessoa com Transtorno do Espectro Autista; e altera o $\S 3^{\circ}$ do art. 98 da Lei $n^{\circ} 8.112$, de 11 de dezembro de 1990. Diário Oficial [da] República Federativa do Brasil, Brasília, DF, 2012.

BRASIL. Ministério da Educação. Lei de Diretrizes e Bases da Educação Nacional. LDB 9.394, de 20 de dezembro de 1996.

BRASIL. Ministério da Educação. Política Nacional de Educação Especial na Perspectiva da Educação Inclusiva e programas. Brasília: MEC, 2008.

CAMARGO, S. P. H.; BOSA, C. A competência social, inclusão escolar e autismo: revisão crítica da literatura. Psicologia \& Sociedade. Universidade Federal do Rio Grande do Sul, Porto Alegre, v. 21, $\mathrm{n}^{\circ}$ 1, p.: 65-74, 2009.

COUTO, M. C. V. et al.. A saúde mental infantil na Saúde Pública brasileira: situação atual e desafios. Revista Brasileira de Psiquiatria. v.30, n 4, p.:390-398, 2008. Disponível em: http://www.scielo. br/pdf/rbp/v30n4/a15v30n4. Acesso em: 10 jun. 2018. 
Uma reflexão sobre as políticas de atendimento para as pessoas

com transtorno do espectro autista

COUTO, M. C. V.. Por uma política pública de saúde mental para crianças e adolescentes. In: FERREIRA, T.(Org). A criança e a saúde mental: enlaces entre a clínica e a política. BH: Autêntica/FHCFUMEC. 2004: 61-74.

DAMASCENO, M. A. et al.. Trabalho do assistente social e a saúde da pessoa com Transtorno do Espectro Autista: contribuições do Serviço Social na garantia do direito na utilização serviços públicos de saúde. ETIC-Encontro de Iniciação Científica. v. 13, n. 13, 2017. Disponível em: file://C:/Users/Alan/ Downloads/6104-16463-1-PB.pdf. Acesso em: 28 nov. 2018.

GUIMARÃES, N.; MARTINS, M.. O autismo na mídia: considerações a respeito dos critérios de noticiabilidade. Cadernos da Escola de Comunicação. n04. p.: 01-09, 2006. Disponível em file:///C:/ Users/Usuario/Downloads/1946-7702-1-PB.pdf. Acesso em: 01 out. 2018.

LEITE, D. S.; HETZEL, S. G. A.. As entidades sociais e a defesa dos direitos das pessoas autistas: dignidade da pessoa humana como pressuposto fundamental de inclusão social. In: Âmbito Jurídico. Rio Grande, XIV, n. 90, jul, 2011. Disponível em: http://www.ambito-juridico.com.br/site/?artigo_id=9880\&n_ link=revista_artigos_leitura. Acesso em: 10 dez. 2018.

MARQUES, M.; DIXE, H. M. A. R.. Crianças e jovens autistas: impacto na dinâmica familiar e pessoal de seus pais. Revista de Psiquiatria Clínica. v.38, n² 2, p.:66-70, 2011. Disponível em: http://www. scielo.br/pdf/rpc/v38n2/v38n2a05. Acesso em: 10 out. 2018.

MATEUS, Manuela Maria Reis. $\mathbf{O}$ assistente social da criança autista e sua família. 2015 . Instituto de Serviço Social do Porto. Tese de Doutorado. Disponível em: https://comum.rcaap.pt/bitstream/10400.26/12352/1/Manuela\%20Maria\%20Reis\%20Mateus.pdf. Acesso em: 23 ago. 2018.

NUNES, D. R. P. M. et al.. Inclusão educacional de pessoas com Autismo no Brasil: uma revisão da literatura. Revista Educação Especial. v. 26,I n. 47, I p. 557-572, set./dez. 2013. Disponível em: https:// periodicos.ufsm.br/educacaoespecial/article/view/10178/pdf. Acesso em: 17 ago. 2018.

OLIVEIRA, B. D. C. et al.. Políticas para o autismo no Brasil: entre a atenção psicossocial e a reabilitação. Physis: Revista de Saúde Coletiva, Rio de Janeiro, v. 27, nº 3, p.:707-726, 2017.

OLIVEIRA, C. Um retrato do autismo no Brasil. Revista Espaço Aberto, USP, nº 170, jun/julho, s/p, 2015.

OLIVEIRA, G. Autismo: diagnóstico e orientação Parte I - Vigilância, rastreio e orientação nos cuidados primários de saúde. Acta Pediátrica Portuguesa. v. 40, n 6, p.: 278-287, 2009. Disponível em: https://www.cpjcoimbra.com/wp-content/uploads/2017/03/Autismo.pdf. Acesso em: 06 ago. 2018.

OLIVEIRA, J. G. C.. Autismo, Política de Saúde Mental e a Psicanálise: (des) Encontros Possíveis. Revista Psicologia \& Saberes, [S.I.], v. 1, n. 1, maio 2012. Disponível em: http://revistas.cesmac.edu.br/ index.php/psicologia/article/view/44. Acesso em: 18 ago. 2018.

ONZI, F. Z.; GOMES, R. F.. Transtorno do Espectro Autista: a importância do diagnóstico e reabilitação. Caderno Pedagógico. Lajeado, v. 12, n. 3, p. 188-199, 2015. Disponível em: http://univates.br/ revistas/index.php/cadped/article/view/979/967. Acesso em: 20 out. 2018.

ORGANIZAÇÃO DAS NAÇÕES UNIDAS (ONU). Convenção sobre os Direitos das Pessoas com Deficiência, 2006. 
ORGANIZAÇÃO DAS NAÇÕES UNIDAS (ONU). Declaração Mundial de Educação para Todos e Plano de Ação para Satisfazer as Necessidades Básicas de Aprendizagem. Conferência Mundial sobre Educação para Necessidades Especiais, 1994. Salamanca-Espanha. Genebra: UNESCO, 1994.

ORGANIZAÇÃO DAS NAÇÕES UNIDAS (ONU). Declaração Mundial de Educação para Todos. Conferência de Jomtien, Tailândia. UNICEF, 1990

RIOS, C. et al. Da invisibilidade à epidemia: a construção narrativa do autismo na mídia impressa brasileira. Revista Interface: Comunicação, Educação e Saúde. v. 19, nº 53, abril-jun., p.:325-335, 2006.

RODRIGUES, I. B. et al.. Análise institucional do discurso de professores de alunos diagnosticados como autistas em inclusão escolar. Revista de Psicologia teoria e prática. vol.14, n. 1 p.: 70-83, 2012. Disponível em: http://pepsic.bvsalud.org/scielo.php?script=sci_arttext\&pid=S1516-36872012000100006\&lng=pt \&nrm=iso. Acesso em: 27 maio 2018.

SILVA, R, M.. Para além do discurso oficial das políticas públicas: possibilidade de (re)pensar o paradigma de inclusão escolar para o educando com transtorno do espectro autista na cidade de Manaus. Manaus: UFAM. Dissertação de Mestrado. 2013. Disponível em: http://200.129.163.131:8080/bitstream/ tede/3187/1/raimunda\%20maria.pdf. Acesso em: 30 set. 2018.

SPÍNOLA. G. O.. Autismo: o ideal e o real na efetivação da decisão jurisdicional que implementa políticas públicas. Revista Brasileira de Políticas Públicas. Brasília, v. 4, n. 1, p.: 55-65, 2014.

TAMANAHA, A. C. et al.. Uma breve revisão histórica sobre a construção dos conceitos do Autismo Infantil e da síndrome de Asperger. Revista Sociedade Brasileira de Fonoaudiologia. v.13, n³. p.:296269, 2008. Disponível em: http://www.scielo.br/pdf/rsbf/v13n3/a15v13n3. Acesso em: 09 set. 2018.

VIEIRA, N. M. et al. Inclusão escolar de alunos com autismo: o que diz a literatura. GT5- Educação, Comunicação e Tecnologia. s/d. p.1-10. Disponível em: http://www.educaretransformar.net.br/wpcontent/uploads/2017/04/INCLUSAO_ESCOLAR_ALUNOS_AUTISMO_QUE_DIZ_LITERATURA.pdf. Acesso em: 29 ago. 2018. 